\title{
EFEITO DO ACOPLAMENTO DOS MODELOS NUMÉRICOS ROMS-WRF NA CAMADA LIMITE PLANETÁRIA - UM ESTUDO DE CASO SOBRE A CONFLUÊNCIA BRASIL-MALVIAS
}

\author{
Gabriel B. Münchow ${ }^{1,3}$, Rita de Cássia M. Alves ${ }^{1}$, Luciano P. Pezzi ${ }^{2}$ \\ ${ }^{1}$ LMQA/CEPSRM/UFRGS - Porto Alegre - Rio Grande do Sul - \\ ${ }^{2} \mathrm{GMO} / \mathrm{CPTEC} / \mathrm{INPE}$ - São José dos Campos - São Paulo \\ 33gabriel.munchow@ufrgs.br
}

\section{RESUMO}

A camada limite planetária (CLP), região da atmosfera na qual habitamos, é fortemente regida pela interação com a superfície terrestre. Neste trabalho foi estudado o efeito na CLP da confluência Brasil-Malvinas utilizando o COAWST, um modelo numérico acoplado oceano-atmosfera-ondas-transporte de sedimentos. Para isso foi realizado dois experimentos, um somente com o modelo COAWST e outro somente o modelo atmosférico WRF. Foi notada uma intensificação nos gradientes horizontais de vento e theta, e no cisalhamento vertical do vento. Estas alterações podem levar a instabilidades afetando níveis mais altos da atmosfera.

\begin{abstract}
The planetary boundary layer (PBL) region of the atmosphere, is strongly influencied by the interaction with the surface. This paper studied the effect on PBL over the ocean using a coupled ocean-atmosphere-wave-sediment transport numerical model (COAWST). It was done two experiments, one with COAWST and another with atmospheric model WRF.
\end{abstract}

\section{INTRODUÇÃO}

A camada limite planetária (CLP), região da atmosfera na qual habitamos, é fortemente regida pela interação com a superfície terrestre (Stull, 2009). É nesta que ocorrem as trocas de energia entre a atmosfera e a superfície, formado por solo, vegetação, oceano, prédios, etc. Para cada caso, há interações e efeitos diferentes na atmosfera. Segundo Pezzi (2005), a 
CLP sobre a Confluência Brasil-Malvinas (CBM) é modulada pelos fortes gradientes de temperatura da superfície do mar (TSM) na região. Neste trabalho, foi estudado o efeito na CLP sobre o oceano, utilizando um modelo numérico acoplado oceano-atmosfera. Para isso, utilizou-se o sistema acoplado Coupled Ocean-Atmosphere-Wave-Sediment Transport Modeling System (COAWST), que possibilita a utilização de modelos atmosféricos, oceânico, ondas e transporte de sedimentos.

\section{METODOLOGIA E RESULTADOS}

O sistema acoplado oceano-atmosfera-ondas-sedimentos COAWST (WARNER et al., 2010) é composto pelo modelo meteorológico Weather Research and Forecasting (WRF) (SKAMAROCK et al., 2005), modelo oceânico Regional Oceanographic Modeling System (ROMS) (SHCHEPETKIN AND MCWILLIAMS, 2005), modelo de ondas Simulating WAves Nearshore (BOOIJ et al., 1999) e o modelo transporte de sedimentos da Community Sediment Transport Modeling Project (WARNER et al., 2008). Neste trabalho, foram utilizados apenas os modelos WRF e ROMS. Foram realizados 2 experimentos, sendo um com o modelo acoplado COAWST (WRF+ROMS), denominado exp1, e somente o modelo atmosférico WRF, denominado exp2.

A região de estudo compreende parte do sul da América do Sul e Oceano Atlântico até $17^{\circ} \mathrm{O}$ de longitude, e a faixa entre $24^{\circ} \mathrm{S}$ e $42^{\circ} \mathrm{S}$ de latitude. Em todos os experimentos, o modelo WRF foi configurado com resolução horizontal de $17 \mathrm{~km}$ e 28 níveis verticais. Foram utilizados como condição inicial (CI) e contorno (CC) atmosférico os dados NCEP/NCAR Global Reanalysis. Para o exp2, também foi utilizado o campo diário de TSM do NCEP/NCAR. A grade oceânica possui resolução horizontal de $1 / 6^{\circ}$ e 40 níveis verticais. As CI e CC são provenientes do projeto Simple Ocean Data Assimilation (CARTON e GIESE, 2008). O período simulado nos experimentos foi de 13 de dezembro de 1979 até dezembro de 1980. Mas neste trabalho foi selecionado o dia 20 de junho de 1980 as 00 e 12 UTC.

A seguir, serão mostrados os resultados dos exp1 e exp2, na Figura 1, na qual é apresentado o corte zonal vertical da atmosfera em $38^{\circ} \mathrm{s}$ juntamente com campos horizontais de TSM e vento em $10 \mathrm{~m}$. No corte vertical, podemos ver em preto a componente zonal do vento, em cinza a componente meridional e em cores a temperatura potencial (theta). É possível notar que em ambos os experimentos, a atmosfera acima de $800 \mathrm{hPa}$ apresenta comportamento 
semelhante. Porém, abaixo desta camada, o efeito da CBM, região com forte gradiente zonal de TSM na Figura 1, é visível. Sobre as águas frias e a CBM há fortes gradientes verticais e horizontais de theta, do litoral argentino até $54,5 \mathrm{~W}$. Nesta região o cisalhamento vertical do vento também é forte e a componente zonal do vento em superfície apresenta valores positivos, enquanto que entre $55 \mathrm{~W}$ e $54,5 \mathrm{~W}$ há valores negativos, gerando uma região com convergência e vorticidade negativa sobre o lado quente da CBM (figura não mostrada). No exp2, o cisalhamento vertical do vento é muito fraco sobre o oceano, assim como os gradientes horizontais de theta. Somente acima de $900 \mathrm{hPa}$ é visto a variação vertical do theta.

COAWST
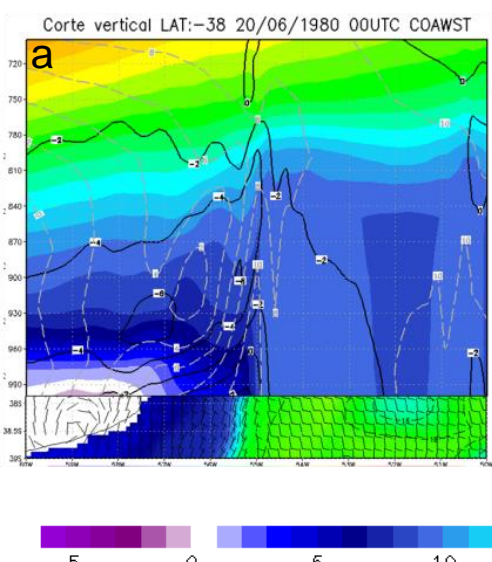

Corte vertical LAT:-38 20/06/1980 12UTC COAWST

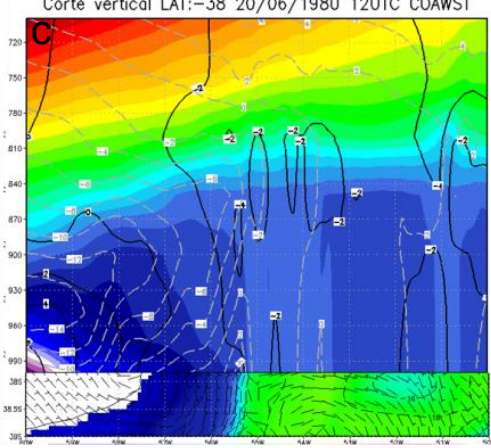

WRF
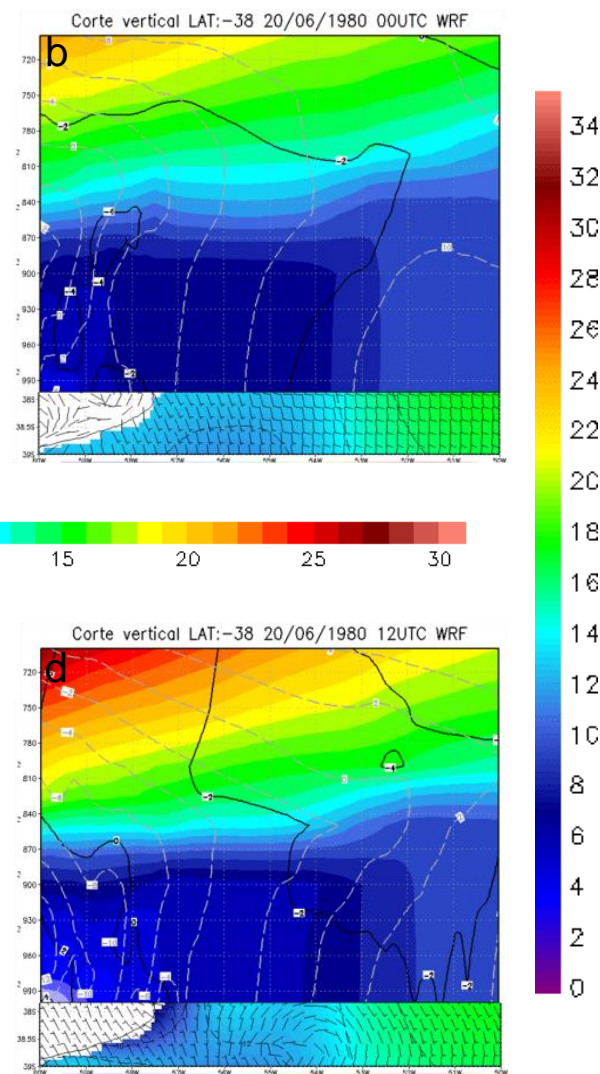

32

14

12

$1 \mathrm{C}$

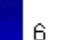

6

4

2
0

Figura 1 - Corte vertical zonal em $38^{\circ} \mathrm{S}, \mathrm{u}(\mathrm{m} / \mathrm{s})$ (linha preta), $\mathrm{v}(\mathrm{m} / \mathrm{s})$ (pontilhado cinza), theta $\left({ }^{\circ} \mathrm{C}\right)$ (sombreado - escala no meio), TSM $\left({ }^{\circ} \mathrm{C}\right)$ (sombreado - escala a esquerda) e vento em $10 \mathrm{~m}(\mathrm{~m} / \mathrm{s})(\mathrm{barb})$. a) exp1 00UTC; b) exp2 00UTC; c) exp1 12UTC; d)exp2 12UTC.

\section{CONCLUSÕES}

Neste trabalho, foi mostrado o impacto de um forte gradiente de TSM, como no caso da 
CBM. Foram verificados gradientes horizontais e verticais de theta mais intensos, assim como cisalhamento do vento. Estas alterações podem levar a instabilidades ou outras alterações na atmosfera, mostrando a importância de estudos específicos sobre esta região utilizando modelo acoplado oceano-atmosfera.

\section{REFERÊNCIAS}

Booij, N., Ris, R.C., Holthuijsen, L.H. A third-generation wave model for coastal regions. Part I: Model description and validation. Journal of Geophysical Research, 104 (C4), 7649-7666, 1999.

Carton, J.; Giese, B. S. A Reanalysis of Ocean Climate Using Simple Ocean Data Assimilation (SODA). Monthly Weather Review, 136, 2999-3017, 2008.

Shchepetkin, A.F., McWilliams, J.C. The Regional Ocean Modeling System: a split-explicit, free-surface, topography-following coordinates ocean model. Ocean Modelling, 9, 347-404, 2005.

Skamarock, W.C., Klemp, J.B., Dudhia, J., Gill, D.O., Barker, D.M., Wang, W., Powers, J.G. A Description of the Advanced Research WRF Version 2. NCARTechnical Note, NCAR/TN-468+STR, 2005.

Warner, J.C., Armstrong, B., He R., Zambon, J.B. Development of a Coupled OceanAtmosphere-Wave-Sediment Transport (COAWST). Modeling System. Ocean Modelling, $35,230-244,2010$.

Warner, J.C., Sherwood, C.R., Signell, R.P., Harris, C., Arango, H.G. Development of a three-dimensional, regional, coupled wave, current, and sediment-transport model.

Computers and Geosciences, 34, 1284-1306, 2008.

Pezzi, L. P., Souza, R.B., Dourado ,M. S., Garcia, C. A. E., Mata , M. M., Silva-Dias, M. A. F. Ocean-atmosphere in situ observations at the BrazilMalvinas Confluence region,

Geophys. Res. Lett., 32, L22603, 2005. 